
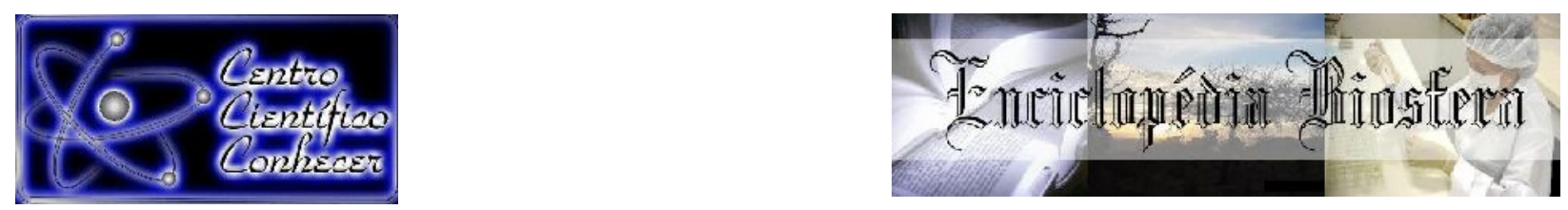

\title{
AVALIAÇÃO DO IMPACTO DO PESO, DA ÁREA DE SUPERFICIE E DO VOLUME CORPORAL EM LEITÕES SOBRE A CAPACIDADE DE SOBREVIVÊNCIA NA FASE DE MATERNIDADE
}

\footnotetext{
Felipe Rômulo Duarte '; Jessica Silva Soares ${ }^{2}$; Robson Carlos Antunes ${ }^{3}$.

${ }^{1}$ Médico Veterinário pela Universidade Federal de Uberlândia- Uberlândia, MG - Brasil

${ }^{2}$ Mestranda em Ciências Veterinárias- Universidade Federal de Uberlândia

(jessica vet@hotmail.com) - Uberlândia, MG - Brasil

${ }^{3}$ Professor Doutor da Universidade Federal de Uberlândia- Uberlândia, MG Brasil
}

Recebido em: 22/09/2018 - Aprovado em: 23/11/2018 - Publicado em: 03/12/2018 DOI: $10.18677 /$ EnciBio_2018B15

\begin{abstract}
RESUMO
Com os avanços nos programas de melhoramento genético as fêmeas suínas tornaram-se hiperprolificas. Embora esta característica resulte em um maior número de leitões desmamados a mortalidade durante a fase de maternidade é preocupante representando grandes perdas para a suinocultura industrial. Objetivou-se com este estudo avaliar a relação entre área de superfície pelo volume do corpo de leitões que morreram esmagados e seus respectivos companheiro de baia. Foi realizado o acompanhamento de fêmeas suínas da linhagem NAIMA ${ }^{\circledR}$ do parto até o desmame para registro de mortes dos leitões por esmagamento. Quando constatada a morte por esmagamento era coletado os dados de comprimento e circunferência para verificar as diferenças entre os leitões que morreram esmagados e seus respectivos companheiros de baia que sobreviveram na mesma leitegada, em que se encontravam no momento da morte. Foram avaliadas 32 leitegadas, um total de 192 leitões dos quais 160 leitões vivos e 32 leitões provenientes de morte por esmagamento. A média dos pesos dos leitões esmagados e a relação área de superfície pelo volume apresentaram diferença estatisticamente significativa. A correlação de Pearson foi significativa para todas as variáveis analisadas e a área de superfície e volume apresentaram correlação positiva com o peso, enquanto a relação área de superfície sobre volume foi inversamente correlacionada com peso e volume corporal.
\end{abstract}

PALAVRAS-CHAVE: leitegada. Mortalidade. Uniformidade.

\section{EVALUATION OF THE IMPACT OF WEIGHT, SURFACE AREA AND BODY VOLUME IN PIGLETS ON SURVIVAL CAPACITY MATERNITY PHASE}

\begin{abstract}
Nowadays, the advances in genetic breeding programs changed the sow lines which have become hyperprolific. Although this characteristic results in a larger number of weaned piglets, the mortality inside the farrow room is some representing large losses for industrial pig farms. The aim of this study was to evaluate the relationship between surface area by the body volume of crushed piglets and their respective littermates. Follow-up of sows from the NAIMA line of birth was
\end{abstract}


carried out until weaning to record the deaths of piglets by crushing. When crush death was found, data on length and circumference were collected to verify the differences between crushed piglets and their respective littermates who survived in the same litter they were at the time of death. Thirty-two litters were evaluated, a total of 192 piglets of which 160 live piglets and 32 piglets coming from death by crushing. The mean weights of the crushed piglets and the surface area ratio by volume presented a statistically significant difference. Pearson correlation was significant for all variables analyzed and surface area and volume presented a positive correlation with weight, while surface area to volume ratio was inversely correlated with body weight and volume.

KEYWORDS: Litter. Mortality. Uniformity.

\section{INTRODUÇÃO}

Com o desenvolvimento dos programas de melhoramento genético a composição genética das fêmeas suínas foi alterada. Passou a ter-se como características primordiais a hiperprolificidade e deposição de carne magra na carcaça (MELLAGI, 2010; MAGNABOSCO, 2016). Embora a seleção para tamanho de leitegada resulte em maior número de leitões desmamados por ano a mortalidade de leitões ainda é preocupante na suinocultura, pois até $20 \%$ dos leitões morrem antes do desmame (OCEPEK et al., 2016; OCEPEK et al., 2017).

O aumento da leitegada está associado com a redução do peso ao nascer e por consequência uma maior variabilidade entre os leitões (ROTWEEL et al., 2012), isso ocorre, porque o crescimento e desenvolvimento fetal tornou-se limitado devido à diminuição do fluxo sanguíneo por feto reduzindo a eficiência placentária (CAMPOS et al., 2012). Entretanto, placentas que apresentam maior vascularização e uma boa angiogênese, aumentam sua eficiência e possibilitam maior transferência de nutrientes e oxigênio para os fetos, proporcionando maior viabilidade dos mesmos (CAMPOS et al., 2012; WIENTJES et al., 2015).

No entanto, o peso não é o único indicador válido de viabilidade, medidas de proporcionalidade corporal são indicadores valiosos do risco de mortalidade (BAXTER et al., 2008; BAXTER et al., 2013). Como a identificação de leitões que apresentem crescimento intrauterino retardado (CIUR) ou seja, aqueles que apresentam peso ao nascer inferior a dois desvios padrões da média do peso corporal para dada idade gestacional são classificados como CIUR (WU et al., 2008). A mortalidade neonatal está relacionada com o peso ao nascer e por consequência com a assincrônia durante o desenvolvimento embrionário e com o aumento da variação de peso e tamanho entres os leitões.

A mortalidade de leitões na maternidade envolve vários fatores entre eles nutrição e saúde da matriz durante a gestação, qualidade e quantidade do leite fornecido aos leitões, peso ao nascer, número de irmãos, número de tetos funcionais, quantidade e qualidade do leite fornecido pela matriz, ambiente (FERREIRA et al., 2014). Fatores estes que resultam principalmente em dois desfechos que são a morte por esmagamento, e morte pelas consequências da diarreia frequentemente encontrada nos leitões (FERREIRA et al., 2014). Em leitões neonatos as principais causas de mortalidade são a não ingestão de colostro, esmagamento pela porca e a dificuldade de termoregulação, essas três causas interagem entre si (ANDERSIN; PEDERSEN, 2016). O tamanho da leitegada pode estar associado com o aumento do risco de mortes por resfriamento, uma vez que esses animais apresentam maior variabilidade (leitões de baixo peso apresentam 
maiores dificuldades termoregulatórias) (RUTHERFORD et al., 2013). Como uma das principais causas que levam ao desfecho da morte do leitão é o esmagamento pela matriz, justifica-se a discussão e a realização de trabalhos científicos sobre este fator.

Objetiva-se com este estudo testar a hipótese de que existe uma relação entre volume do corpo e área de superfície de leitões que morreram esmagados diferentes entres os que sobreviveram da mesma baia. Relação esta que favorece a perda de calor nos que morreram por esmagamento.

\section{MATERIAL E MÉTODOS}

\section{Local e animais}

A coleta de dados foi realizada em uma granja comercial de suínos no município de Uberlândia - MG. Foi utilizada a linhagem fêmea NAIMA para a coleta de dados. A matriz prenhe foi alojada em gaiolas individuais que disponha de um bebedouro tipo chupeta e comedouro tipo calha. Aos 110 dias de gestação a matriz foi transferida para a sala maternidade onde ficou até o desmame. Foi realizado o acompanhamento dessas matrizes do parto ao desmame para verificar a ocorrência de mortes dos leitões por esmagamento. Quando constatada a morte por esmagamento era coletado os dados para verificar as diferenças entre os leitões que morreram esmagados e seus companheiros de baia que sobreviveram na mesma leitegada em que se encontravam no momento da morte.

Foram avaliadas 32 leitegadas, um total de 192 leitões dos quais 160 leitões vivos e 32 leitões provenientes de morte por esmagamento. Os animais foram divididos em grupo 1- leitões esmagados e grupo 2-leitões vivos.

\section{Pesagem, medidas de comprimento e circunferência}

A pesagem de todos os leitões foi realizada com a balança eletrônica portátil fabricada pela Walmur Instrumentos Veterinários Ltda®, tipo gancho com precisão de 20 gramas. Com o auxílio de uma corda os leitões foram amarrados e pendurados à balança e posteriormente foi registrado o peso de cada um.

O comprimento de cada leitão foi aferido com fita métrica fabricada pela Círculo S.A., graduada em milímetros de 1,5 metros, o comprimento foi aferido na região dorsal a partir da inserção do pescoço (articulação atlanto occipital) até a base da cauda. A circunferência do leitão foi aferida logo abaixo das axilas abrangendo as regiões dorsal e ventral com o auxílio da mesma fita métrica. Foram coletados os dados dos leitões mortos por esmagamento e de todos os seus respectivos companheiros de baia, uma vez que a granja adotava o manejo de remanejar os leitões para uniformizar as leitegadas. Assim nem todos os leitões da baia são irmãos.

Com os dados de comprimento e perímetro da circunferência foram calculados a área de superfície onde considera-se o corpo dos leitões como um cilindro perfeito, sendo a base uma circunferência perfeita e a altura o comprimento. Para isto, foi calculado o raio, volume do cilindro, área da base e área do corpo. Após realizado o cálculo da área de superfície e volume foi realizada a relação entre essas duas variáveis. 
Raio (r)

$$
\mathrm{r}=\frac{\text { circunferência }}{2 * \pi}
$$

Volume do cilindro (vc)

$$
\mathrm{vc}=\pi * \mathrm{r}^{2} \mathrm{x} \text { altura }
$$

\section{Área de superfície do cilindro}

$$
\begin{aligned}
& \text { área da base }=\pi * \mathrm{r}^{2} \\
& \text { área do corpo }=\text { perímetro da base * altura } \\
& \text { área de superficíe }=2 \text { * área da base }+ \text { área do corpo }
\end{aligned}
$$

\section{Relação: área de superfície e volume de cada leitão}

$$
\text { relação }=\frac{\text { área de superficie }}{\text { volume }}
$$

Todas as equações para cálculos geométricos estão descritas no livro Geometria Analítica e Álgebra Linear (LIMA, 2005).

\section{ANÁLISE ESTÁTISTICA}

As variáveis (área de superfície, volume, relação entre superfície e volume e peso) do grupo 1 e grupo 2 foram submetidas ao teste de Anderson-Darling. Posteriormente foi aplicado o teste $t$ de student para comparação das médias. Foi realizada análise de correlação linear de Pearson entre as variáveis para verificar a existência de correlação entre elas. Todas as análises foram feitas no software Action com nível de significância menor ou igual a $5 \%(P \leq 0,05)$.

\section{RESULTADOS E DISCUSSÕES}

A média dos pesos dos leitões esmagados foi menor $(P=0,047)$ que dos sobreviventes (Tabela 1). Ser mais leve resulta em dificuldades na competição pelos tetos nos momentos das mamadas, esse leitão tende a estar mal alimentado, apresentando apatia e dificuldades em controlar sua temperatura corporal que é agravada pela imaturidade etária (BAXTER et al., 2008; FURTADO, 2012).

O peso ao nascer é considerado um indicador crítico do desempenho pósnatal, uma vez que leitões que nascem abaixo do peso possuem desenvolvimento retardado ao longo da vida (FIX et al., 2010; DOUGLAS et al., 2013). Nos primeiros dias de vida animais com baixo peso ao nascer apresentam menores reservas corporais quantidades baixas de energia e por consequência dificuldades para mamar, competição entre os irmãos e dificuldade em manter a temperatura corporal (DOUGLAS et al., 2013).

Galiot et al. (2018), acompanharam os leitões do nascimento até o desmame e constatou que $43,2 \%$ das mortes ocorreram por esmagamento concluindo que 0 peso ao nascer e o ganho de peso nas primeiras 24 horas são os principais fatores que influenciam o crescimento e a mortalidade pré-desmama do leitão. 
Como em outros estudos, o baixo peso ao nascer foi um preditor confiável de mortalidade pré-desmame (BAXTER et al., 2008; FIX et al., 2010). Pois estes animais apresentam menores reservas energéticas o que dificulta a capacidade de regulação da temperatura corporal (GALIOT et al., 2018), uma vez que o peso ao nascer tem uma correlação positiva com a temperatura retal (ANDERSEN; PEDERSEN, 2016).

A média das áreas de superfície $(P=0,049)$ e volumes $(P=0,044)$ foram menores nos leitões esmagados. A relação entre a área de superfície e volume foi maior nos esmagados $(P=0,050)$, o que indica que os leitões esmagados apresentam área de superfície maior em relação ao volume do que seus respectivos companheiros de baia que estavam vivos até o momento da coleta. Assim há uma maior facilidade em perder calor e maior dificuldade em controlar sua temperatura corporal (AIRES et al., 2014). Provavelmente estes leitões apresentam maiores quedas de temperatura corporal, procuram se aquecer próximo à mãe e são esmagados pela mesma. Uma vez que a temperatura retal nas primeiras $24 \mathrm{~h}$ são preditores de sobrevida (GALIOT et al., 2018).

A seleção genética para o tamanho de leitegada resultou em leitões mais heterogêneos, com um maior número de animais menores levando a uma menor viabilidade ao nascimento (PANZARDI et al., 2013; PANDOLFI et al., 2017). A capacidade prejudicada de leitões menores em manter o equilíbrio homeotérmico é explicada pela sua maior área de superfície o que resulta em uma maior perda de calor, além disso o peso corporal também influencia na capacidade de termoregulação (HERPIN et al., 2002; ANDERSON; PEDERSON, 2016).

TABELA 1- Peso, área de superfície, volume e ralação: área de superfície/volume dos leitões esmagados e leitões vivos. Resultados compostos de média, +/- desvio padrão e (erro padrão).

\begin{tabular}{|c|c|c|c|c|}
\hline & Peso & $\begin{array}{l}\text { Área de } \\
\text { superfície }\end{array}$ & Volume & $\begin{array}{l}\text { Relação: } \\
\text { superfície } \\
\text { volume }\end{array}$ \\
\hline $\begin{array}{l}\text { Leitões } \\
\text { esmagados }\end{array}$ & $\begin{array}{c}1,44 \mathrm{~kg} \\
+/-0,47 \mathrm{~kg} \\
(0,08)\end{array}$ & $\begin{array}{c}779,5 \mathrm{~cm}^{2} \\
+/-201,4 \mathrm{~cm}^{2} \\
(35,64)\end{array}$ & $\begin{array}{c}1390,9 \mathrm{~cm}^{3} \\
+/-548,6 \mathrm{~cm}^{3} \\
(97,09)\end{array}$ & $\begin{array}{c}0,59 \\
+/-0,09 \\
(0,015)\end{array}$ \\
\hline $\begin{array}{l}\text { Leitões } \\
\text { vivos }\end{array}$ & $\begin{array}{c}1,75 \mathrm{~kg} \\
+/-0,43 \mathrm{~kg} \\
(0,034)\end{array}$ & $\begin{array}{c}878,3 \mathrm{~cm}^{2} \\
+/-192,8 \mathrm{~cm}^{2} \\
(15,3) \\
\end{array}$ & $\begin{array}{c}1675 \mathrm{~cm}^{3} \\
+/-560,5 \mathrm{~cm}^{3} \\
(44,48)\end{array}$ & $\begin{array}{c}0,55 \\
+/-0,07 \\
(0,005) \\
\end{array}$ \\
\hline P-valor & 0,047 & 0,049 & 0,044 & 0,050 \\
\hline
\end{tabular}

A correlação de Pearson foi significativa para todas as variáveis analisadas. As variáveis área de superfície e volume apresentaram correlação positiva com o peso. A relação área de superfície/volume apresentou correlação negativa, indicando que quanto maior a superfície menor o volume do leitão (Tabela 2). Ou seja, leitões menores possuem maior área de superfície, isto implica na termoregulação do leitão nas primeiras horas de vida (PANDOLFI et al., 2017; GALIOT et al., 2018).

Uma vez que leitões com baixo peso ao nascer possuem menores reservas de energia e são mais vulneráveis a queda de temperatura resultando em 
dificuldade em acessar as tetas da porca para mamar o colostro e maior risco de morte por esmagamento (GALIOT et al., 2018). Como demostrado por Baxter et al., (2008), leitões que morreram durante a fase maternidade possuíam temperatura retal menor do que aqueles que atingiram a fase de desmama. Portanto não é surpreendente que a relação área de superfície/volume quando relacionado com mortes por esmagamento seja uma correlação negativa.

TABELA 2- Correlação de Pearson grupo 1- leitões esmagados e grupo 2leitões vivos.

\begin{tabular}{c|c|c|c|c}
\hline & Peso & $\begin{array}{c}\text { Área de } \\
\text { superfície }\end{array}$ & Volume & $\begin{array}{c}\text { Relação: } \\
\text { superfície/volume }\end{array}$ \\
\hline Peso & - & 0,95 & 0,95 & $-0,93$ \\
\hline $\begin{array}{c}\text { Área de } \\
\text { superfície }\end{array}$ & $*$ & - & 0,99 & $-0,97$ \\
\hline Volume & $*$ & $*$ & - & $-0,96$ \\
\hline $\begin{array}{c}\text { Relação: } \\
\text { superfície/volume }\end{array}$ & $*$ & $*$ & $*$ & - \\
$\begin{array}{l}\text { * Os valores de P são todos 0,00000 indicando correlações altamente significativas. Acima da } \\
\text { diagonal encontram-se os valores de correlação e abaixo a significância dos mesmos. }\end{array}$
\end{tabular}

\section{CONCLUSÃO}

O presente estudo confirmou que o peso ao nascer é uma das principais variáveis que determina o crescimento e a sobrevivência dos leitões na fase de maternidade. Também mostrou que os leitões que morreram esmagados apresentavam maior área de superfície em relação ao volume corporal em comparação aos seus respectivos companheiros da baia, comprovando assim que estes animais possuem uma maior dificuldade de termoregulação o que resulta em maior número de mortes por esmagamento.

\section{REFERÊNCIAS}

AIRES, J. F.; METZ, M.; BIRCK, L. J. Causas de mortalidade de leitões até o desmame em granja comercial na região noroeste do Rio Grande do Sul: Relatório técnico-científico. 2014. Disponível em: $<$ https://www.revistas.unijui.edu.br/index.php/salaoconhecimento/article/download/34 99/2899>.

ANDERSEN, I. L.; VASDAL, G.; and PEDERSEN, L. J. Nest building and posture changes and activity budget of gilts housed in pens and crates. Applied Animal Behaviour Science, v.159, p.29-33, $2014 . \quad$ Disponível em:<https://www.appliedanimalbehaviour.com/article/S0168-1591(14)001853/fulltext>. doi:10.1016/j.applanim.2014.07.002

BAXTER, E. M.; JARVIS, S.; D'EATH. R. B.; ROSS, D. W.; ROBSON. S. K. etal. Investigating the behavior al and physiological indicators of neonatal survival in pigs. Theriogenology, v.69, p.773-783, 2008. Disponível em: <http://dx.doi.org/10.1016/j.theriogenology.2007.12.007>. doi:10.1016/j.theriogenology.2007.12.007

BAXTER, E. M.; RUTHERFORD, K. M. D.; D'EATH, R. B.; ARNOTT, G.; TURNER, 
S. P. et al. The welfare implications of large litter size in the domestic pig II: management factors. Animal Welfare, v.22,p.219-238, 2013. Disponível em:<http://dx.doi.org/10.7120/09627286.22.2.219>.

doi: $10.7120 / 09627286.22 .2 .219$

CAMPOS, P. H. R. F.;SILVA, B. A. N. J.; DONZELE, L.; OLIVEIRA, R. F. M.; and $\mathrm{KNOL}$, E. F. Effects of sow nutrition during gestation on within-litter birth weight variation: A review. Animal, v.6, p.797-806, 2012. Disponível em:<https://www.ncbi.nlm.nih.gov/pubmed/22558927>. doi:10.1017/S1751731111002242

FERREIRA, A. H.; CARRARO, B.; DALLANORA, D. Produção de Suínos: Teoria e Prática. Brasília: Abcs, 2014. 905 p.

DOUGLAS, S. L.; EDWARDS, S. A.; SUTCLIFFE, E.; KNAP, P. W.; AND KYRIAZAKIS, I. Identification of risk factors associated with poor lifetime growth performance in pigs. Journal animal science, v.91, p.4123-4132, 2013. Disponivél em:<https://www.ncbi.nlm.nih.gov/pubmed/23825336>

doi:10.2527/jas2012-5915

FIX, J. S.; CASSADY, J. P.; HERRING, W. O.; HOLL, J. W.; CULBERTSON, M. S. et al. Effect of piglet birth weight on body weight, growth, back fat, and longissimus muscle area of commercial market swine. Livestock Science, v.127, p.51-59, 2010. Disponível em:<https://www.sciencedirect.com/science/article/pii/S1871141309002996>. doi:10.1016/j.livsci.2009.08.007

FURTADO, C. S. D.; MELLAGI, A. P. G.; CYPRIANO, C. R.; GAGGINI, T. S.; MARI LOURDES BERNARDI, M. L. et al. Influência do Peso ao Nascimento e de Lesões Orais, Umbilicais ou Locomotoras no Desempenho de Leitões Lactentes. Acta Scientiae Veterinarrie, v.40, Issue 4, p.1-7, $2012 . \quad$ Disponível em:<http://www.ufrgs.br/actavet/40-4/040-4.htm

GALIOT, L.; LACHANCE, I.; LAFOREST, JEAN-PAUL.; GUAY, F. Modelling piglet growth and mortality on commercial hog farms using variables describing individual animals, litters, sows and management factors. Animal Reproduction Science, v.188, p. 57-65, $2018 . \quad$ Disponível em:<https://www.sciencedirect.com/science/article/pii/S0378432017305341?via\%3Di hub>doi: 10.1016/j.anireprosci.2017.11.009

LIMA, E. L. Geometria Analítica e Álgebra Linear. Rio de Janeiro; IMPA, 2005

MAGNABOSCO, D.; BERNARDI, M.; WENTZ, I.; CUNHA, E.; BORTOLOZZO, F. Low birth weight affects lifetime productive performance and longevity of female swine. Livestock Science, v.184, p. 119-125, 2016. Disponível em:<https://www.sciencedirect.com/science/article/pii/S1871141315300615>. doi:10.1016/j.livsci.2015.12.008

MELLAGI, A. P.; ARGENTI, L. E.; FACCIN, J. E. G.; BERNARDI, M. L.; WENTZ, I. et 
al. Aspectos nutricionais de matrizes suínas durante a lactação e o impacto na fertilidade. Acta Scientiae Veterinariae, v. 38, n. Supl 1, p. 181-209, 2010. Disponível em:<https://www.researchgate.net/publication/272834956_Aspectos_nutricionais_de _matrizes_suinas_durante_a_lactacao_e_o_impacto_na_fertilidade>.

OCEPEK, M.; ANDERSEN-RANBERG, I.; EDWARDS, S. A.; FREDRIKSEN, B.; FRAMSTAD, T. et al. Can a super sow be a robust sow? Consequences of litter investment in purebred and crossbred sows of different parities. Journal Animal science, v.95, p. 3550-3560, 2016. Disponível em <https://brage.bibsys.no/xmlui/handle/11250/2463122> doi:10.2527/jas.2016-0386

OCEPEK, M.; ROSVOLD, E. M.; ANDERSEN-RANBERG, I.; AND ANDERSEN, I. L. Can we improve maternal care in sows? Maternal behavioral traits important for piglet survival in loose-housed sow herds. Journal Animal Science, v.95, p. 47084717, 2017. Disponível em<https://academic.oup.com/jas/articleabstract/95/11/4708/4807424?redirectedFrom=fulltext> doi:10.2527/jas2017.1725

PANZARDI, A.; BERNARDI, M.L.; MELLAGI, A.P.; BIERHALS, T.; BORTOLOZZO, F.P. et al. Newborn piglet traits associated with survival and growth performance until weaning. Preventive Veternary Medicine, v.110, p. 206-213, 2013. Disponível em<https://www.sciencedirect.com/science/article/pii/S0167587712003935?via\%3Di hub>doi:10.1016/j.prevetmed.2012.11.016

PANDOLFI, S.; EDWAERDS, S. A.; ROBERT, F.; KYRIAZAKIS, I. Risk factors associated with the different categories of piglet perinatal mortality in French farms. Preventive Veternary Medicine, v. 137, Part A, p. 1-12, 2017. Disponível em:<https://www.sciencedirect.com/science/article/pii/S0167587716306493?via\%3Di hubs.

doi:10.1016/j.prevetmed.2016.12.005

HERPIN, P.; JEAN LE DIVIDICH, M. D. Development of thermoregulation and neonatal survival in pigs. Livestock Production Science, v.78, p. 25-45, 2002. Disponível em:<https://www.sciencedirect.com/science/article/pii/S0301622602001835?via\%3Di hub>doi:10.1016/S0301-6226(02)00183-5

ROOTWELT, V.; REKSEN, O.; FARSTAD, W.; AND FRAMSTAD, T. Postpartum deaths: Piglet, placental, and umbilical characteristics. Journal Animal Science, v.91, p. 2647-2656, 2012. Disponível em< https://academic.oup.com/jas/article/91/6/2647/4717172> doi:10.2527/jas.2012-5531

RUTHERFORD, K.; BAXTER E.; D'EATH, R.; TURNER, S.; ARNOTT, G. et al. The welfare implications of large litter size in the domestic pig I: biological factors. Animal Welfare, v.22, p.199-218, $2013 . \quad$ Disponível em<http://www.ingentaconnect.com/content/ufaw/aw/2013/00000022/00000002/art0 
0006>.

doi:10.7120/09627286.22.2.199

WIENTJES, J. G. M.; SOEDE, N. M.; KNOL, E. F.; VAN DEN BRAND, H.; KEMP, B. Piglet birth weight and litter uniformity: Effects of weaning-to-pregnancy interval and body condition changes in sows of different parities and crossbred lines. Journal of Animal Science, v.91, p.2099-2107, 2015. Disponível em: $<$ https://www.ncbi.nlm.nih.gov/pubmed/23463562>. doi:10.2527/jas.2012-5659

WU, G.; BAZER, F.W.; DATTA, S.; JOHNSON, G.A.; LI, P.; et al. Proline metabolism in the conceptus: Implications for fetal growth and development. Amino Acids, v.35, p.691-702, 2008.

Disponível em:<https://link.springer.com/article/10.1007\%2Fs00726-008-0052-7>. doi: $10.1007 /$ s00726-008-0052-7 